\title{
After trees die: quantities and determinants of necromass across Amazonia
}

\author{
K.-J. Chao ${ }^{1,2}$, O. L. Phillips ${ }^{1}$, T. R. Baker ${ }^{1}$, J. Peacock ${ }^{1}$, G. Lopez-Gonzalez ${ }^{1}$, R. Vásquez Martínez ${ }^{3}$, \\ A. Monteagudo ${ }^{3,4}$, and A. Torres-Lezama ${ }^{5}$ \\ ${ }^{1}$ Earth and Biosphere Institute, School of Geography, University of Leeds, LS2 9JT, Leeds, UK \\ ${ }^{2}$ International Master Program of Agriculture, College of Agriculture and Natural Resources, National Chung Hsing \\ University, Taichung 40227, Taiwan \\ ${ }^{3}$ Proyecto Flora del Perú, Jardín Botánico de Missouri, Oxapampa, Peru \\ ${ }^{4}$ Herbario Vargas, Universidad Nacional San Antonio Abad del Cusco, Cusco, Peru \\ ${ }^{5}$ INDEFOR, Facultad de Ciencias Forestales y Ambientales, Universidad de Los Andes, Mérida, Venezuela
}

Received: 3 December 2008 - Published in Biogeosciences Discuss.: 12 February 2009

Revised: 5 June 2009 - Accepted: 26 June 2009 - Published: 11 August 2009

\begin{abstract}
The Amazon basin, one of the most substantial biomass carbon pools on earth, is characterised by strong macroecological gradients in biomass, mortality rates, and wood density from west to east. These gradients could affect necromass stocks, but this has not yet been tested. This study aims to assess the stocks and determinants of necromass across Amazonian forests. Field-based and literature data were used to find relationships between necromass and possible determinants. Furthermore, a simple model was applied to estimate and extrapolate necromass stocks across terra firma Amazonian forests. In eight northwestern and three northeastern Amazonian permanent plots, volumes of coarse woody debris $(\geq 10 \mathrm{~cm}$ diameter) were measured in the field and the density of each decay class was estimated. Forest structure and historical mortality data were used to determine the factors controlling necromass. Necromass is greater in forests with low stem mortality rates (northeast) rather than in forests with high stem mortality rates (northwest) (58.5 \pm 10.6 and $27.3 \pm 3.2 \mathrm{Mg} \mathrm{ha}^{-1}$, respectively). Using all published necromass values, we find that necromass across terra firma forests in Amazonia is positively related to both forest dynamics (mortality mass inputs and a surrogate for decomposition rate (average wood density of living trees)) and forest structure (biomass), but is better explained by forest dynamics. We propose an improved method to estimate necromass for plots where necromass has not been measured. The estimates, together with other actual mea-
\end{abstract}

Correspondence to: K.-J. Chao (kjungchao@dragon.nchu.edu.tw) surements of necromass, were scaled-up to project a total Amazonian necromass of $9.6 \pm 1.0 \mathrm{Pg} C$. The ratio of necromass (on average weighted by forest region) to coarse aboveground biomass is 0.127. Overall, we find (1) a strong spatial trend in necromass in parallel with other macroecological gradients and (2) that necromass is a substantial component of the carbon pool in the Amazon.

\section{Introduction}

Coarse woody debris (CWD) is a crucial component of forest structure, as it constitutes an aboveground pool of carbon and nutrients (Harmon et al., 1986). CWD can account for 6 to $25 \%$ of total aboveground vegetative mass (biomass plus necromass) in the neotropics (Nascimento and Laurance, 2002; Delaney et al., 1998; Rice et al., 2004), but these studies are limited in their spatial extent. For example, in Amazonia, the world's largest tropical forest which is responsible for $\sim 50 \%$ of the biome's productivity and respiration, necromass reports have been biased to forests in eastern Amazonia, which are known to be one extreme of Amazonian forest types in terms of both structure and function (Malhi et al., 2006; Phillips et al., 2004). There are only two studies of CWD stocks (necromass) (Chao et al., 2008a; Baker et al., 2007) and one of CWD volume (Gale, 2000) from western forests, and there has been little pan-Amazon evaluation of necromass stocks (e.g., Saatchi et al., 2007). In the absence of direct measurements, necromass is often estimated as a fixed percentage of biomass (e.g., Houghton et al., 2001; Malhi et al., 2006; Saatchi et al., 2007), assuming

Published by Copernicus Publications on behalf of the European Geosciences Union. 
that higher biomass forests should also accrue more necromass. However, the relationship between necromass and biomass has not yet been adequately demonstrated in Amazonia, nor have other potential determinants (e.g., mortality mass-inputs or decomposition rate) been properly considered. A comparison of CWD stocks in regions with contrasting biomass stocks and forest dynamics would help to reveal their determinants.

Factors that potentially control necromass include forest type, structure, and successional stage (Harmon et al., 1986), but must ultimately be related to the balance of mortality inputs and decomposition outputs (forest dynamics) (Olson, 1963). Quantitative studies of mortality input in mass and CWD decay rates in the tropics are few (e.g., Chambers et al., 2000; Carey et al., 1994). However, some other strong macroecological gradients across Amazonia have been shown: there is a two-fold increase in tree stem mortality rates from east to west (Phillips et al., 2004), with smaller concomitant decreases in biomass and wood densities (Baker et al., 2004; Malhi et al., 2006). It may therefore be expected that there are also differences in necromass stocks, too.

The questions we asked in this study are: (1) is there variation in the quantity of necromass across Amazonia? (2) can necromass be predicted from forest structural parameters (biomass) or dynamic parameters (mortality measures and decomposition estimates) of a stand? We hypothesised that forest dynamics, rather than forest structure, determine necromass stocks. First, we predicted $\left(P_{1.1}\right)$ that there is no relationship between stocks of biomass and necromass, contrary to a basic assumption made in many carbon budget studies. Our second prediction $\left(P_{2.1}\right)$ is that forests with high mass-mortality rates (amount of dead mass) and slow decomposition rates have higher stocks of necromass. We also expected $\left(P_{2.2}\right)$ that forest stands with higher wood density would have lower decay rates, and thus higher necromass stocks, as decomposition rate is negatively correlated with wood density (Chambers et al., 2000).

We explore these ideas using our field measurements, published data, and a tree-by-tree census dataset (the RAINFOR project; Malhi et al., 2002; Peacock et al., 2007). Firstly, volumes of CWD were measured using the plot-based method (Harmon and Sexton, 1996) in two regions of Amazonia, and densities of CWD estimated using equations developed by Chao et al. (2008a). Secondly we examined the relationships between necromass and forest structure and dynamics (i.e., mortality and a decomposition surrogate) using our field results and published data. Finally, a simple model was applied to predict CWD stocks for places where necromass has not been measured, assuming a steady state (Olson, 1963). A fully pan-Amazonian perspective on necromass stock was developed by combining existing measurements with the predicted values.

\section{Methods}

\subsection{CWD stocks: data sources}

There are three types of data: measured necromass based on our field work (hereafter termed as field-based), measured necromass based on literature (literature), and estimated necromass based on census data (estimated).

Field-based necromass measurements were conducted in two regions of mature terra firma Amazonian forests: one in the northwest (NW, eight ca. 1-ha plots), and the other in the northeast (NE, three 0.5-ha plots). NW Amazonia plots (ALP-A, ALP-B, SUC-01, SUC-02, SUC-04, SUC-05, YAN-01 and YAN-02) were located in northern Peru (Allpahuayo, $3^{\circ} 57^{\prime} \mathrm{S}, 73^{\circ} 26^{\prime} \mathrm{W}$; Sucusari, $3^{\circ} 26^{\prime} \mathrm{S}$, $72^{\circ} 54^{\prime} \mathrm{W}$; Yanamono $3^{\circ} 26^{\prime} \mathrm{S}, 72^{\circ} 51^{\prime} \mathrm{W}$ ) (Vásquez Martínez and Phillips, 2000; Vásquez Martínez, 1997). The NE Amazonian plots are located at El Dorado (ELD-01/02 and $-03 / 04,6^{\circ} 05-06^{\prime} \mathrm{N}, 61^{\circ} 24^{\prime} \mathrm{W}$ ) and Rio Grande (RIO-01/02, $8^{\circ} 06^{\prime} \mathrm{N}, 61^{\circ} 41^{\prime} \mathrm{W}$ ), Venezuela (Veillon, 1985). Since the establishment of these plots, living trees with a diameter $\geq 10 \mathrm{~cm}$ have been tagged, identified, and measured at approximately four to five year intervals (Malhi et al., 2002).

Literature necromass values incorporated published results from humid, lowland Amazonian forests. We used both the literature and our field-based necromass values to explore pan-Amazonian relationships between forest structure and dynamic parameters and necromass.

Estimated necromass values were obtained for plots in the RAINFOR database (Peacock et al., 2007) where stocks of necromass have not been measured. The chosen plots were restricted to those located in terra firma Amazonian forests that have been recently recensused (between 2000 and 2006) (Appendix A). The estimation method was based on the relationship between decay rate and wood density and a simple steady state model (Olson, 1963). The estimated necromass values, together with measured CWD values (field-based and literature), were used to extrapolate necromass stocks across Amazonia.

\subsection{CWD stocks: field-based measurement}

CWD stock (necromass, $\mathrm{Mg} \mathrm{ha}^{-1}$ ) for decay class $d(d=1$ to 3 ) is the product of volume $(v)$ and density $\left(\rho_{d}\right)$, and then standardised by the size of the plot. In our field work, we measured the volume of and classified decay classes for all dead woody material, including trees, lianas, and palms, with a diameter $\geq 10 \mathrm{~cm}$.

CWD volume within a plot was measured by the plotbased method (Harmon and Sexton, 1996) in 2004 for the NE plots and in 2005 for the NW plots. Both CWD lying on the ground (fallen CWD) and standing and broken stumps (standing CWD) were included. Diameter $1\left(D_{1}\right)$ and $2\left(D_{2}\right)$ at each end of a CWD piece were measured to the nearest centimeter. For logs tapering to less than $10 \mathrm{~cm}$ diameter, 
diameters and lengths were taken to where the piece reached $10 \mathrm{~cm}$. When wood was partially buried in litter and therefore difficult to measure, diameters were taken as two perpendicular cross-sections of the wood, one horizontal and one vertical to the ground. Diameters of standing CWD were measured at the lowest part of the trunk above any buttress roots. The diameter of the narrower end of a stump was taken from the fallen log on the ground, and where this was not possible it was visually estimated. Major attached branches of standing trees were visually estimated. Where CWD was hollow, the thickness of the solid section was recorded and used to adjust the volume of CWD. The volume $\left(v, \mathrm{~m}^{3}\right)$ of each CWD piece was calculated using Smalian's formula (Phillip, 1994):

$v=L_{\mathrm{CWD}}\left[\frac{\pi\left(D_{1} / 2\right)^{2}+\pi\left(D_{2} / 2\right)^{2}}{2}\right]$

where $L_{\mathrm{CWD}}(\mathrm{m})$ is the length of a CWD piece, and $D$ is the diameter $(\mathrm{m})$ at either end. If two measurements (horizontal and vertical to the ground) were taken, the geometric mean was used to estimate diameter. For hollow CWD pieces, volume was calculated by subtracting the inner void volume from the outer volume.

Decay classes $(d)$ of CWD were classified in the field, including intact (class $1, d=1$ ), partially decayed (class $2, d=2$ ), and rotten (class 3, $d=3$ ) as described in Chao et al. (2008a). Where the decay classes of bark and heartwood were very different, classes were assigned separately. In humid, lowland neotropical forests, density of each CWD decay class $\left(\rho_{d}, \mathrm{~g} \mathrm{~cm}^{-3}\right)$ is closely related to the plot-level living wood density (Chao et al., 2008a). Thus, $\rho_{d}$ was estimated as a function of the plot-average wood density of live trees.

$\rho_{d=1}=1.17\left[\rho_{B A j}\right]-0.21$

and

$\rho_{d=2}=1.17\left[\rho_{B A j}\right]-0.31$

where $\rho_{d=1}$ and $\rho_{d=2}$ represent the CWD densities in decay class one $(d=1)$ and two $(d=2)$, respectively, and $\rho_{B A} j$ $\left(\mathrm{g} \mathrm{cm}^{-3}\right)$ is the wood density of living trees of plot $j$, weighted by their basal area. For CWD in decay class three, the average value of density for debris in "decay class three" from published studies of humid, lowland neotropical forests $\left(0.29 \mathrm{~g} \mathrm{~cm}^{-3}\right)$ was used, as suggested by Chao et al. (2008a). The living wood density $\left(\rho_{B A}\right)$ of plot $j$ were obtained from the RAINFOR database (Peacock et al., 2007) and a species wood density database (Chave et al., 2006; López-Gonzalez et al., 2006; Baker et al., 2004). The use of the three decay class method is less susceptible to potential problems of small sample sizes and the high diversity of tropical trees than a five decay class method (Chao et al., 2008a, cf., Keller et al., 2004; Palace et al., 2007; Rice et al., 2004). Wood density data were matched to plot data on a tree-by-tree basis. In cases where species-level wood densities were unavailable, the average for the genus (34\% of 5401 individuals) or family $(5 \%)$ was used. For unidentified trees and individuals where family-level data were lacking (2\%), the average wood density of all stems in the plot was used.

\subsection{CWD determinants: CWD input and decay rate}

CWD input (annual mortality mass input, $I$, $\mathrm{Mgha}^{-1}$ year $^{-1}$ ) for each plot is the sum of dead tree biomass $\left(\mathrm{AGB}_{\text {coarse}}\right.$, see next section), calculated using prior-to-death diameter measures and the allometric models (Chambers et al., 2001; Chave et al., 2005), divided by the census interval. We used short interval (about 4-year) census data to represent recent mortality events ( $I_{\text {recent }}$, $\mathrm{Mg} \mathrm{ha}^{-1}$ year $^{-1}$ ).

As the decomposition rate is a function of wood density (Fig. 1a in Chambers et al., 2000), we used $\rho_{B A}\left(\mathrm{~g} \mathrm{~cm}^{-3}\right)$ as a surrogate for the plot-level decomposition rate.

\subsection{CWD determinants: coarse aboveground biomass}

Aboveground biomass as dry weight (AGB, $\mathrm{kg}$ ) of each plot was estimated. A locally-derived AGB allometric model is currently unavailable for our studied regions, so we applied two models developed from other tropical forests. The first model is the Chambers model (Chambers et al., 2001), based on harvesting at one site near Manus, Brazil (Higuchi et al., 1998), derived from trees larger than $5 \mathrm{~cm}$ in diameter at $1.3 \mathrm{~m}$ or above the buttresses $(n=315)$. This model was adjusted to account for species-level wood density as suggested by Baker et al. (2004):

$$
\begin{aligned}
\mathrm{AGB} & =\left[\frac { \rho _ { i } } { 0 . 6 7 } \operatorname { e x p } \left(0.333\left[\ln D_{i}\right]+0.933\left[\ln D_{i}\right]^{2}\right.\right. \\
& \left.\left.-0.122\left[\ln D_{i}\right]^{3}-0.370\right)\right]
\end{aligned}
$$

where $\rho_{i}\left(\mathrm{~g} \mathrm{~cm}^{-3}\right)$ is the species-level wood density of tree $i$, and $D_{i}(\mathrm{~cm})$ is the diameter at $1.3 \mathrm{~m}$ of the same tree.

The second model is the Chave model, from a pan-tropical, multi-site $(n=15)$ study of "moist forests" with allometric models derived from trees larger than $5 \mathrm{~cm}$ (Chave et al., 2005). The Chave model accounts for species-level wood density:

$$
\begin{aligned}
\mathrm{AGB} & =\rho_{i} \exp \left(2.148\left[\ln D_{i}\right]+0.207\left[\ln D_{i}\right]^{2}\right. \\
& \left.-0.0281\left[\ln D_{i}\right]^{3}-1.499\right)
\end{aligned}
$$

Our preliminary comparisons showed that estimates using the Chave model were generally greater than those based on the Chambers model, especially for trees larger than $80 \mathrm{~cm}$. We report estimates using both models to represent a possible range of AGB for our plots.

Coarse aboveground biomass $\left(\mathrm{AGB}_{\text {coarse }}, \mathrm{Mg}\right.$ ) was estimated by multiplying AGB with a correction factor (0.85) to account for the proportion of biomass in branches $\geq 10 \mathrm{~cm}$ 
Table 1. Necromass and coarse aboveground biomass in the northwestern (NW) and northeastern (NE) Amazonia (average \pm 1 SE).

\begin{tabular}{|c|c|c|c|c|c|c|}
\hline \multirow[t]{2}{*}{ Region } & \multirow[t]{2}{*}{ Year } & \multicolumn{4}{|c|}{ Necromass (N) $\left(\mathrm{Mg} \mathrm{ha}^{-1}\right)$} & \multirow[t]{2}{*}{$\mathrm{S} / \mathrm{F}^{*}$} \\
\hline & & Intact & Partially decayed & Rotten & Total & \\
\hline NW & 2005 & $10.1 \pm 1.5$ & $15.2 \pm 2.6$ & $2.0 \pm 0.5$ & $27.3 \pm 3.2$ & $0.34 \pm 0.07$ \\
\hline $\mathrm{NE}$ & 2004 & $18.4 \pm 7.1$ & $39.0 \pm 8.7$ & $1.1 \pm 0.6$ & $58.5 \pm 10.6$ & $0.43 \pm 0.22$ \\
\hline
\end{tabular}

\begin{tabular}{llllll}
\hline (b) Biomass & \multicolumn{5}{l}{} \\
\hline \multirow{2}{*}{ Region } & Year & \multicolumn{3}{c}{ Biomass $\left(\mathrm{AGB}_{\text {coarse }}\right)\left(\mathrm{Mg} \mathrm{ha}^{-1}\right)^{\dagger}$} & \multicolumn{2}{c}{ N/AGB coarse $^{\ddagger}$} \\
\cline { 3 - 6 } & & Chambers & Chave & Chambers & Chave \\
\hline NW & 2001 & $247.9 \pm 1.9$ & $266.7 \pm 2.5$ & $10.3 \pm 1.3$ & $11.1 \pm 1.3$ \\
NW & 2005 & $253.6 \pm 3.1$ & $274.6 \pm 4.8$ & $10.1 \pm 1.3$ & $10.9 \pm 1.4$ \\
\hline NE & 2000 & $344.5 \pm 58.4$ & $376.4 \pm 76.5$ & $16.8 \pm 4.5$ & $18.0 \pm 4.4$ \\
NE & 2004 & $337.3 \pm 59.4$ & $368.6 \pm 75.9$ & $17.3 \pm 4.8$ & $18.5 \pm 4.8$ \\
\hline
\end{tabular}

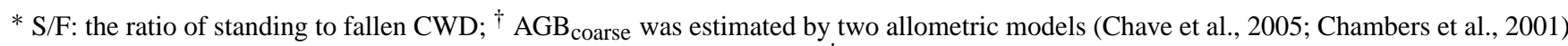
$\sim 4$ years prior to the CWD census and in the year of CWD census itself; ${ }^{\ddagger} N / \mathrm{AGB}_{\text {coarse }}(\%)$ : the ratio of total necromass to coarse biomass.

diameter only (Higuchi, unpublished data, cited in Chambers et al., 2000).

\subsection{CWD estimation}

For places where necromass has not been measured, a simple model (Olson, 1963; see also Keller et al., 2004; Palace et al., 2007, 2008) was applied to predict CWD stocks in the Discussion Sects. 4.3 and 4.4. This is based on the following equation,

$d N / d t=I-k_{S S} N$

where $k_{s s}$ is the decomposition rate at steady state (year ${ }^{-1}$ ), $I$ is the mortality mass input $\left(\mathrm{Mg} \mathrm{ha}^{-1}\right.$ year $\left.^{-1}\right)$ of that plot, and $N$ is necromass $\left(\mathrm{Mg} \mathrm{ha}^{-1}\right)$. Assuming forests are close to dynamic equilibrium (steady state), the change of necromass $(\mathrm{d} N / \mathrm{d} t)$ would be equal to zero. Therefore, decay rates of CWD can be expressed as

$k_{s s}=I / N$

As the decay rate is negatively correlated with wood density (Fig. 1a in Chambers et al., 2000), we sought a relationship between $\rho_{B A j}\left(\mathrm{~g} \mathrm{~cm}^{-3}\right)$ and $k_{s s}$ (based on the literature and our field-based values for necromass and mortality mass input). Such a relationship would allow necromass to be estimated using mortality mass data $(I)$ and living wood density ( $\rho_{B A j}, k_{s s}$ surrogate), as $N=I / k_{s s}$.

\section{Results}

\subsection{Stocks of measured CWD}

In our field study, total necromass (stock of CWD) was greater in the northeastern than in the northwestern plots (Mann-Whitney U test, $p=0.025$ ) (Table 1). In both regions, most CWD was partially decayed (Table 1). The ratio of standing to fallen CWD is 0.34 in the NW and 0.43 in the NE plots (Table 1). Taking CWD into account increased coarse aboveground vegetative mass by up to $11 \%$ in NW Amazonia and $19 \%$ in NE Amazonia, compared with aboveground live biomass alone $\left(N / \mathrm{AGB}_{\text {coarse }}\right.$, Table 1$)$.

The average $( \pm 1 \mathrm{SE})$ necromass of available Amazonian studies is $31.6 \pm 2.7\left(\mathrm{Mg} \mathrm{ha}^{-1}\right)$ in terra firma, 23.4 \pm 7.4 in white sand forests, and $10.4 \pm 2.5$ in floodplain forests (Table 2). The necromass measures of terra firma Amazonian forests will be referred to as literature necromass values hereafter.

\subsection{Determinants of measured CWD across terra firma Amazonian forests}

We found positive relationships between biomass $\left(\mathrm{AGB}_{\text {coarse }}\right)$ and necromass (from field-based and literature measures, Table 2) (Fig. 1a):

$N=0.133\left[\mathrm{AGB}_{\text {coarse }}\right]-1.678\left(r^{2}=0.124, p=0.038\right)(8)$

Also, necromass is positively related to recent mortality mass input ( $\left.I_{\text {recent }}\right)$ (Fig. 1b),

$N=5.237\left[I_{\text {recent }}\right]+4.061\left(r^{2}=0.234, p=0.007\right)$

and plot-level average living wood density (Fig. 1c):

$N=161.451\left[\rho_{B A}\right]-70.474\left(r^{2}=0.368, p<0.001\right)(10)$ 
Table 2. Coarse woody debris across humid, lowland Amazonian forests, including terra firma, sandy forest, and floodplain types from this study and other publications. D., minimum diameter criteria of coarse woody debris $(\mathrm{cm})$; Volume $\left(V, \mathrm{~m}^{3} \mathrm{ha}^{-1}\right) ; \mathrm{Necromass}\left(N, \mathrm{Mg} \mathrm{ha}^{-1}\right.$, $\pm 1 \mathrm{SE}) ; \mathrm{S} / \mathrm{F}$, ratio of standing to fallen CWD; $\mathrm{AGB}_{\text {coarse }}$, coarse aboveground biomass $\left(\mathrm{Mg} \mathrm{ha}^{-1}\right.$, aboveground biomass multiplied by coarse correction factor, 0.85$) ; I_{\text {recent }}$, recent mortality mass input $\left(\mathrm{Mg} \mathrm{ha}^{-1}\right.$ year $\left.^{-1}\right) ; \rho_{B A} j$, average living wood density of plot $j$, weighted by basal area $\left(\mathrm{g} \mathrm{cm}^{-3}\right)$.

\begin{tabular}{|c|c|c|c|c|c|c|c|c|c|c|}
\hline Forest type* & Region $^{\dagger}$ & Plot Name & D. & Volume & Necromass & $\mathrm{S} / \mathrm{F}$ & AGB $_{\text {coarse }}$ & $I_{\text {recent }}$ & $\rho_{B A j^{\#}}$ & Reference \\
\hline terra firma & NE & ELD-01/02 & 10 & 118.31 & $74.5( \pm-, \mathrm{a})$ & 0.27 & $434.5^{\S}$ & 6.7 & 0.769 & This study \\
\hline terra firma & $\mathrm{NE}$ & ELD-03/04 & 10 & 137.36 & $62.8( \pm-, a)$ & 0.16 & $229.7^{\S}$ & 8.6 & 0.648 & This study \\
\hline terra firma & $\mathrm{NE}$ & RIO-01/02 & 10 & 72.62 & $38.4( \pm-, a)$ & 0.87 & $347.8^{\S}$ & 6.3 & 0.708 & This study \\
\hline terra firma & $\mathrm{NE}$ & $\begin{array}{l}\text { ELD-01/02 and } \\
\text { RIO-01/02 }\end{array}$ & 2.5 & - & $33.3( \pm 7.5, a)$ & 0.80 & 303.7 & $2.6^{\#}$ & 0.740 & Delaney et al. (1998) \\
\hline terra firma & $\mathrm{NE}$ & $\begin{array}{l}\text { San Carlos de } \\
\text { Rio Negro }\end{array}$ & 7.6 & - & $23.1( \pm 10.3, b)$ & - & - & - & - & Kauffman et al. (1988) \\
\hline terra firma & $\mathrm{NE}$ & $\begin{array}{l}\text { San Carlos de } \\
\text { Rio Negro }\end{array}$ & 7.6 & - & $7.6( \pm 4.9, \mathrm{~b})$ & - & - & - & - & Kauffman et al. (1988) \\
\hline terra firma & NW & $\begin{array}{l}\text { Upper Rio } \mathrm{Ne}- \\
\text { gro }\end{array}$ & 5 & - & $26.3( \pm 9.1, a)$ & - & 187.9 & - & - & Saldarriaga et al. (1988) \\
\hline terra firma & NW & ALP-A & 10 & 71.62 & $31.4( \pm-, \mathrm{a})$ & 0.16 & $254.7^{\S}$ & 2.5 & 0.649 & This study \\
\hline terra firma & NW & ALP-B & 10 & 94.55 & $41.1( \pm-, a)$ & 0.71 & $235.3^{\S}$ & 7.9 & 0.617 & This study \\
\hline terra firma & NW & SUC-01 & 10 & 54.70 & $21.5( \pm-, a)$ & 0.20 & $251.0^{\S}$ & 5.9 & 0.593 & This study \\
\hline terra firma & NW & SUC-02 & 10 & 62.57 & $27.4( \pm-, a)$ & 0.10 & $251.1^{\S}$ & 4.4 & 0.614 & This study \\
\hline terra firma & NW & SUC-04 & 10 & 55.81 & $25.5( \pm-, a)$ & 0.43 & $260.0^{\S}$ & 5.5 & 0.623 & This study \\
\hline terra firma & NW & SUC-05 & 10 & 92.92 & $37.9( \pm-, a)$ & 0.35 & $253.3^{\S}$ & 4.8 & 0.607 & This study \\
\hline terra firma & NW & YAN-01 & 10 & 42.05 & $15.4( \pm-, a)$ & 0.24 & $263.0^{\S}$ & 4.8 & 0.560 & This study \\
\hline terra firma & NW & YAN-02 & 10 & 44.87 & $18.6( \pm-, a)$ & 0.52 & $260.4^{\S}$ & 4.1 & 0.593 & This study \\
\hline terra firma & NW & JEN-11 & 10 & 46.60 & $20.3( \pm-, a)$ & 0.41 & $254.8^{\S}$ & 4.6 & 0.669 & Chao et al. (2008a) \\
\hline terra firma & SW & CUZ-01 & 10 & 49.52 & $19.8( \pm-, a)$ & - & $226.0^{\#, \S}$ & $4.9^{\#}$ & 0.581 & Baker et al. (2007, raw data) \\
\hline terra firma & SW & CUZ-02 & 10 & 60.58 & $23.9( \pm-, a)$ & - & $193.6^{\#, \S}$ & $5.2^{\#}$ & 0.513 & Baker et al. (2007, raw data) \\
\hline terra firma & SW & CUZ-03 & 10 & 41.66 & $16.6( \pm-, a)$ & - & $199.4^{\#, \S}$ & $4.6^{\#}$ & 0.566 & Baker et al. (2007, raw data) \\
\hline terra firma & SW & CUZ-04 & 10 & 52.76 & $21.0( \pm-, a)$ & - & $240.8^{\#, \S}$ & $7.4^{\#}$ & 0.586 & Baker et al. (2007, raw data) \\
\hline terra firma & SW & TAM-01 & 10 & 35.04 & $13.5( \pm-, a)$ & - & $201.9^{\#, \S}$ & $3.7^{\#}$ & 0.527 & Baker et al. (2007, raw data) \\
\hline terra firma & SW & TAM-02 & 10 & 81.20 & $33.1( \pm-, a)$ & - & $210.6^{\#, \S}$ & $4.8^{\#}$ & 0.539 & Baker et al. (2007, raw data) \\
\hline terra firma & SW & TAM-04 & 10 & 22.76 & $9.8( \pm-, a)$ & - & $249.5^{\#, \S}$ & $5.3^{\#}$ & 0.618 & Baker et al. (2007, raw data) \\
\hline terra firma & SW & TAM-05 & 10 & 35.63 & $14.3( \pm-, a)$ & - & $215.2^{\#, \S}$ & $4.7^{\#}$ & 0.606 & Baker et al. (2007, raw data) \\
\hline terra firma & SW & TAM-06 & 10 & 15.76 & $6.3( \pm-, a)$ & - & $219.9^{\#, \S}$ & $3.5^{\#}$ & 0.506 & Baker et al. (2007, raw data) \\
\hline terra firma & SW & TAM-07 & 10 & 37.76 & $14.9( \pm-, \mathrm{a})$ & - & $223.6^{\#, \S}$ & $3.9^{\#}$ & 0.579 & Baker et al. (2007, raw data) \\
\hline terra firma & SW & TAM-08 & 10 & 51.84 & $21.6( \pm-, a)$ & - & $188.8^{\#, \S}$ & $3.0^{\#}$ & 0.598 & Baker et al. (2007, raw data) \\
\hline terra firma & $\mathrm{E}$ & Rondônia & $? 10$ & - & $30.0( \pm-, b)$ & - & 242.3 & - & $0.760^{-}$ & Brown et al. (1995) \\
\hline terra firma & $\mathrm{E}$ & Rondônia & 2.5 & - & $30.5( \pm 6.9, \mathrm{~b})$ & - & 260.8 & - & - & Cummings et al. (2002) \\
\hline terra firma & $\mathrm{E}$ & $\begin{array}{l}\text { Juruena, Mato } \\
\text { Grosso, }\end{array}$ & 10 & - & $43.2( \pm 1.6, a)$ & 0.14 & 223.6 & $7.9^{\mathrm{qI}}$ & - & Palace et al. (2007) \\
\hline terra firma & $\mathrm{E}$ & $\begin{array}{l}\text { TUF1, Tapajós } \\
\text { UF, Pará }\end{array}$ & 10 & 94.60 & $52.8( \pm 14.9, \mathrm{a})$ & $0.17^{\mid}$ & 239.7 & $7.9^{\mathrm{ql}}$ & 0.691 & Keller et al. (2004) \\
\hline terra firma & $\mathrm{E}$ & $\begin{array}{l}\text { TUF2, Tapajós } \\
\text { UF, Pará }\end{array}$ & 10 & 94.20 & $51.8( \pm 10.1, a)$ & $0.17^{\mid}$ & 239.7 & $7.9^{\mathrm{qI}}$ & 0.691 & Keller et al. (2004) \\
\hline terra firma & $\mathrm{E}$ & $\begin{array}{l}\text { CUF1, Cauaxi } \\
\text { UF, Pará }\end{array}$ & 10 & 86.60 & $43.8( \pm 12.0, b)$ & - & - & - & 0.691 & Keller et al. (2004) \\
\hline terra firma & $\mathrm{E}$ & $\begin{array}{l}\text { CUF2, Cauaxi } \\
\text { UF, Pará }\end{array}$ & 10 & 97.10 & $52.8( \pm 14.3, \mathrm{~b})$ & - & - & - & 0.691 & Keller et al. (2004) \\
\hline terra firma & $\mathrm{E}$ & $\begin{array}{l}\text { Tapajós UF, } \\
\text { Pará }\end{array}$ & 10 & - & $52.4( \pm 2.4, a)^{+}$ & 0.17 & 239.7 & $8.5^{\mathrm{q}}$ & - & Palace et al. (2007) \\
\hline ? terra firma & $\mathrm{E}$ & $\begin{array}{l}\text { Paragominas, } \\
\text { Pará }\end{array}$ & 10 & - & $55.0( \pm 7.5, a)$ & 0.67 & 219.3 & - & - & Gerwing (2002) \\
\hline ? terra firma & $\mathrm{E}$ & $\begin{array}{l}\text { Vitoria Ranch, } \\
\text { Pará }\end{array}$ & 7.61 & - & $42.3( \pm 19.7, \mathrm{~b})$ & - & - & - & - & Uhl and Kauffman (1990) \\
\hline terra firma & $\mathrm{E}$ & Tapajós, Pará & 10 & 166.70 & $\begin{array}{l}86.6( \pm 13.4 \\
(95 \% \mathrm{CI}), \mathrm{a})\end{array}$ & - & $250.6^{\S}$ & 4.8 & 0.691 & Rice et al. (2004) \\
\hline terra firma & $\mathrm{E}$ & Manaus & 10 & - & $21.0( \pm-, \mathrm{c})$ & - & $310.2^{\#, \S}$ & 3.6 & 0.703 & Chambers et al. (2000) \\
\hline terra firma & $\mathrm{E}$ & $\begin{array}{l}\text { BIONTE, } \\
\text { Manaus }\end{array}$ & $?$ & - & $\begin{array}{l}29.7( \pm 12.2 \\
(? \mathrm{SD}), \mathrm{c})\end{array}$ & - & $310.2^{\#, \S}$ & $2.3^{\#}$ & 0.703 & $\begin{array}{l}\text { Summers (1998) cited in } \\
\text { Chambers et al. (2000) }\end{array}$ \\
\hline terra firma & $\mathrm{E}$ & $\begin{array}{l}\text { Reserva Flo- } \\
\text { restal Adolfo } \\
\text { Ducke, Manaus }\end{array}$ & 3 & - & $9.5( \pm-, b)$ & - & - & - & - & $\begin{array}{l}\text { Martius and Bandeira } \\
(1998)\end{array}$ \\
\hline
\end{tabular}


Table 2. Continued.

\begin{tabular}{|c|c|c|c|c|c|c|c|c|c|c|}
\hline Forest type* & Region $^{\dagger}$ & Plot Name & D. & Volume & Necromass & $\mathrm{S} / \mathrm{F}$ & $\mathrm{AGB}_{\text {coarse }}$ & $I_{\text {recent }}$ & $\rho_{B A j^{\#}}$ & Reference \\
\hline terra firma & E & Manaus & $?$ & & $25.8( \pm-, a)$ & 0.42 & $-!$ & - & - & Klinge (1973) \\
\hline terra firma & $\mathrm{E}$ & Manaus & 10 & - & $31.0( \pm 2.5, a)$ & 0.25 & 276.7 & - & - & $\begin{array}{l}\text { Nascimento and } \\
\text { Laurance (2002) }\end{array}$ \\
\hline Average & & & & $\begin{array}{l}68.5 \\
( \pm 6.6)\end{array}$ & $\begin{array}{l}31.6 \\
( \pm 2.7)\end{array}$ & $\begin{array}{l}0.36 \\
( \pm 0.05)\end{array}$ & $\begin{array}{l}249.1 \\
( \pm 7.9)\end{array}$ & $\begin{array}{l}5.2 \\
( \pm 0.3)\end{array}$ & $\begin{array}{l}0.63 \\
( \pm 0.01)\end{array}$ & \\
\hline White sand & $\mathrm{NE}$ & SCR-04D & 10 & 32.18 & $16.2( \pm-$, a) & 0.43 & $290.8^{\#, \S}$ & - & 0.701 & $\begin{array}{l}\text { Chao et al., } \\
\text { unpublished data }\end{array}$ \\
\hline White sand & $\mathrm{NE}$ & SCR-05D & 10 & 75.87 & $39.8( \pm-$, a) & 0.52 & $295.1^{\#, \S}$ & - & 0.721 & $\begin{array}{l}\text { Chao et al., } \\
\text { unpublished data }\end{array}$ \\
\hline White sand & NE & $\begin{array}{l}\text { San Carlos de } \\
\text { Rio Negro }\end{array}$ & 7.6 & - & $2.5( \pm 1.6, b)$ & - & - & - & - & Kauffman et al. (1988) \\
\hline White sand & NW & ALP-30 & 10 & 76.93 & $37.1( \pm-, a)$ & 0.91 & $233.9^{\#, \S}$ & 3.3 & 0.660 & $\begin{array}{l}\text { Chao et al., } \\
\text { unpublished data }\end{array}$ \\
\hline White sand & NW & JEN-12 & 10 & 86.00 & $41.1( \pm-, \mathrm{a})$ & 0.47 & $236.6^{\#, \S}$ & 0.6 & 0.699 & Chao et al. (2008a) \\
\hline Sandy & $\mathrm{E}$ & $\begin{array}{l}\text { Maracá Island, } \\
\text { Roraima }\end{array}$ & 5 & - & $3.93( \pm-$, a) & 0.33 & - & - & - & Scott (1992) \\
\hline Average & & & & $\begin{array}{l}67.7 \\
( \pm 12.1)\end{array}$ & $\begin{array}{l}23.4 \\
( \pm 7.4)\end{array}$ & $\begin{array}{l}0.53 \\
( \pm 0.10)\end{array}$ & $\begin{array}{l}264.1 \\
( \pm 16.7)\end{array}$ & $\begin{array}{l}2.0 \\
( \pm 1.4)\end{array}$ & $\begin{array}{l}0.70 \\
( \pm 0.01)\end{array}$ & \\
\hline Floodplain & NW & $\begin{array}{l}\text { floodplain plot, } \\
\text { Jenaro Herrera }\end{array}$ & 10 & 42.30 & $10.3( \pm-, b)$ & - & $214.8^{\#, \S}$ & - & 0.510 & Chao et al. (2008a) \\
\hline Floodplain & NW & SUC-03 & 10 & 37.29 & $21.0( \pm-, \mathrm{a})$ & 0.27 & $284.9^{\#, \S}$ & 3.4 & 0.718 & $\begin{array}{l}\text { Chao et al., } \\
\text { unpublished data }\end{array}$ \\
\hline Floodplain & E & $\begin{array}{l}\text { Lago Cobra } \\
23-25 \text { m a.s.1., } \\
\text { Manaus }\end{array}$ & $?$ & - & $3.6( \pm-, c)$ & - & - & 6.0 & - & Martius (1997) \\
\hline Floodplain & E & $\begin{array}{l}\text { Lago Cobra } \\
25-26 \text { m a.s.1., } \\
\text { Manaus }\end{array}$ & $?$ & - & $10.4( \pm-, c)$ & - & - & - & - & Martius (1997) \\
\hline Floodplain & E & $\begin{array}{l}\text { Lago Cobra } \\
26-27 \text { m a.s.1., } \\
\text { Manaus }\end{array}$ & $?$ & - & $5.9( \pm-, c)$ & - & - & - & - & Martius (1997) \\
\hline Floodplain & E & $\begin{array}{l}\text { Lago Central } \\
23-25 \text { m a.s.1., } \\
\text { Manaus }\end{array}$ & $?$ & - & $11.4( \pm-, c)$ & - & - & - & - & Martius (1997) \\
\hline Average & & & & $\begin{array}{l}39.8 \\
( \pm 2.5)\end{array}$ & $\begin{array}{l}10.4 \\
( \pm 2.5)\end{array}$ & - & $\begin{array}{l}249.9 \\
( \pm 35.1)\end{array}$ & $\begin{array}{l}4.7 \\
( \pm 1.3)\end{array}$ & $\begin{array}{l}0.61 \\
( \pm 0.10)\end{array}$ & \\
\hline
\end{tabular}

* terra firma is defined as humid, lowland forest, presumed not to have experienced fluvial flooding in at least 250 years (Phillips et al., 2004), and not on white sand soils; ${ }^{\dagger}$ NE: north-eastern (Venezuela, Guyana, Suriname, and French Guiana); NW: north-western (Columbia, Ecuador, and northern Peru); SW: south-western (Acre state of Brazil and southern Peru); E: eastern (Brazil, excluding Acre); $\ddagger$ Types of CWD, a: includes both fallen and standing CWD; b: only fallen CWD; c: unclear; ${ }^{\circledR} \mathrm{AGB}_{\text {coarse }}$ : aboveground biomass (AGB) estimated by the Chambers model (Eq. 4) multiplied by coarse correction factor, 0.85; I Estimated using standing to fallen CWD ratio in Palace et al. (2007); ${ }^{+}$Not included for Fig. 1, as more detailed results are presented in TUF1 and TUF2 of the Keller et al. (2004) study; II estimated from decomposition rate, assuming in steady state; ${ }^{\#}$ calculated in this study, using adjacent plots from the RAINFOR database (Baker et al., 2004; López-Gonzalez et al., 2006; Chave et al., 2006; Peacock et al., 2007); ${ }^{-}$Average of 13 most dominant trees, weighted by volume; ! The biomass listed in the paper $(731.7 \mathrm{Mg} / \mathrm{ha})$ is fresh weight including epiphytes, saprophytes and lianas, so it is not included in the analysis.

\section{Discussion}

This study presents new measurements of CWD from two regions with a two-fold difference in mortality stem rates, and also shows how pan-Amazonian patterns of CWD vary with other parameters.

\subsection{Stocks and determinants of measured CWD across terra firma Amazonian forests}

Based on our field data, stocks of CWD within regions are higher in NE Amazonia than in NW Amazonia. In other Amazonian studies, coarse necromass ranges from $2.5 \mathrm{Mg} \mathrm{ha}^{-1}$ in a dry and poor-nutrient white sand forest in Venezuela (Kauffman et al., 1988) to $86.6 \mathrm{Mg} \mathrm{ha}^{-1}$ in an oldgrowth Brazilian terra firma forest that was recovering from 
(a)

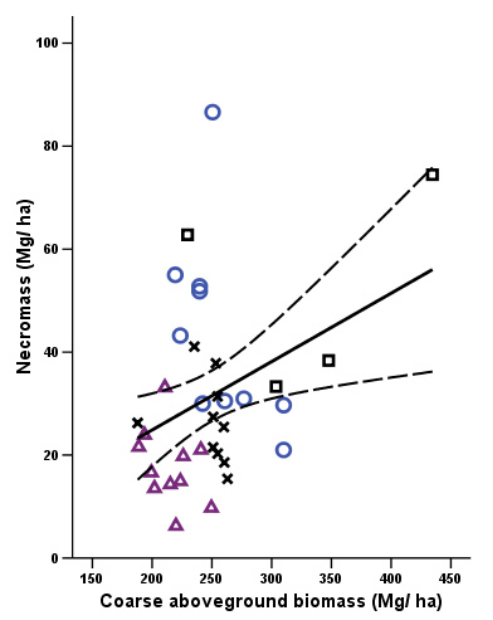

(b)

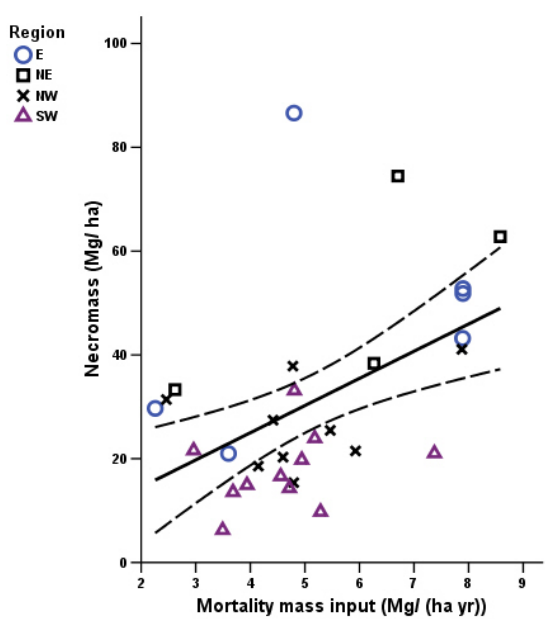

(c)

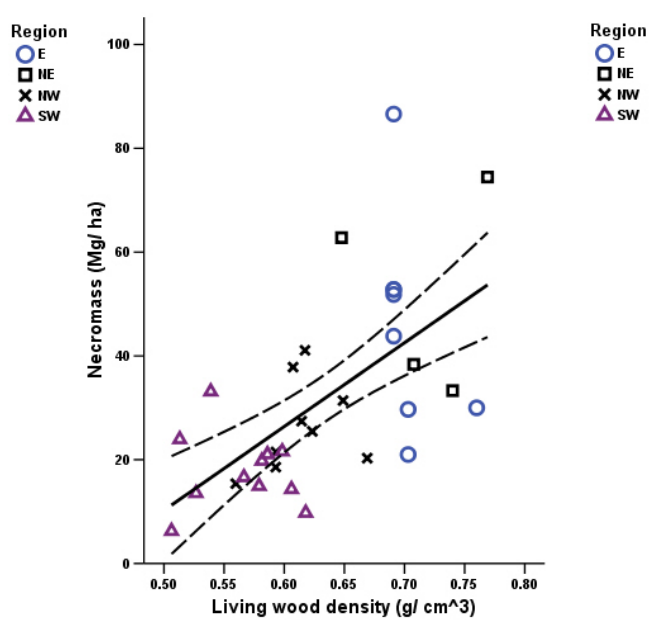

Fig. 1. Relationships between necromass and (a) coarse aboveground biomass, (b) mortality mass input, and (c) plot-average living wood density across terra firma Amazonian forests. Solid lines are regression lines for the model and the dashed lines are the $95 \%$ confidence intervals for means. Relationships between necromass and coarse aboveground biomass $\left(\mathrm{AGB}_{\text {coarse }}\right)\left(r^{2}=0.124, p=0.038\right)$, mortality mass input $\left(I_{\text {recent }}\right)\left(r^{2}=0.234, p=0.007\right)$, and plot-average living wood density $\left(\rho_{B A}\right)\left(r^{2}=0.368, p<0.001\right)$ are all significant. All sites in Table 2 with appropriate data are plotted.

a period of high mortality (Rice et al., 2004). The NE Amazonian forests in our study are towards the higher end of the range, whereas the NW forests are close to the average for terra firma forests. Although the stem mortality rate (number of dead trees) in NE Amazonia was low (Chao et al., 2008b), there was high mortality mass input (amount of dead mass) (Table 2). This pattern suggests that big trees died in the NE forests (see also Chao et al., 2008b) and necromass is closely related to mass-mortality rates rather than stemmortality rates.

When using a larger sample of published terra firma studies, we find positive relationships between necromass and biomass $\left(\mathrm{AGB}_{\text {coarse }}\right)$, mortality mass input $\left(I_{\text {recent }}\right)$, and plotlevel average living wood density $\left(\rho_{B A}\right)$. These findings suggest that across Amazonian forests there is a gradient in necromass that relates to the macroecological gradients in Amazonian forests (see Baker et al., 2004; Malhi et al., 2006). These results do not support our prediction $\left(P_{1.1}\right)$ that there is no relationship between stocks of necromass and biomass, but support the predictions $\left(P_{2.1}\right.$ and $\left.P_{2.2}\right)$ that necromass is positively related to mass-mortality rates and plot-level wood density. However, Fig. 1a showed that among Amazonian forests with similar quantities of biomass, there is a wide range of necromass, suggesting that biomass is less useful than using wood density or mortality mass input to predict necromass. Here, we propose a new hypothesis (modified from our original hypothesis) that necromass is better explained by forest dynamics than by forest structure.

\subsection{Uncertainties}

Some factors which can influence the necromass pool have not been considered in our study. These include: (1) small branch $(<10 \mathrm{~cm}$ diameter) contributions to the necromass pool, (2) branchfall contributions to necromass production, and (3) temporal variation in mortality rates.

Small branches $(2-10 \mathrm{~cm}$ diameter) may contribute a significant portion to total necromass pool (e.g., $8-18 \%$ in Keller et al., 2004; 10\% in Rice et al., 2004), but we did not take this portion of dead wood into consideration as there are few appropriate data.

Necromass production (mortality mass input) in our study is estimated by mortality census data, but Palace et al. (2008) stressed that this method can underestimate necromass production by up to $30-50 \%$. This is mainly due to overlooking that branchfall can contribute considerably to the flux (e.g., Clark et al., 2001). Better quantification of the necromass production in branchfall would reduce the uncertainties of the relationship between necromass production and pool size.

Our calculations are based on the assumption that the studied forests are in dynamic equilibrium. Our studied plots are located in forests free of cyclones, but wind-storms or droughts sometimes affect forests. For example, the extreme value of CWD reported from Tapajos (Rice et al., 2004), is likely to reflect an earlier large disturbance. Census interval length may also influence the mortality mass input. For example, longer periods may lead to an underestimation of 


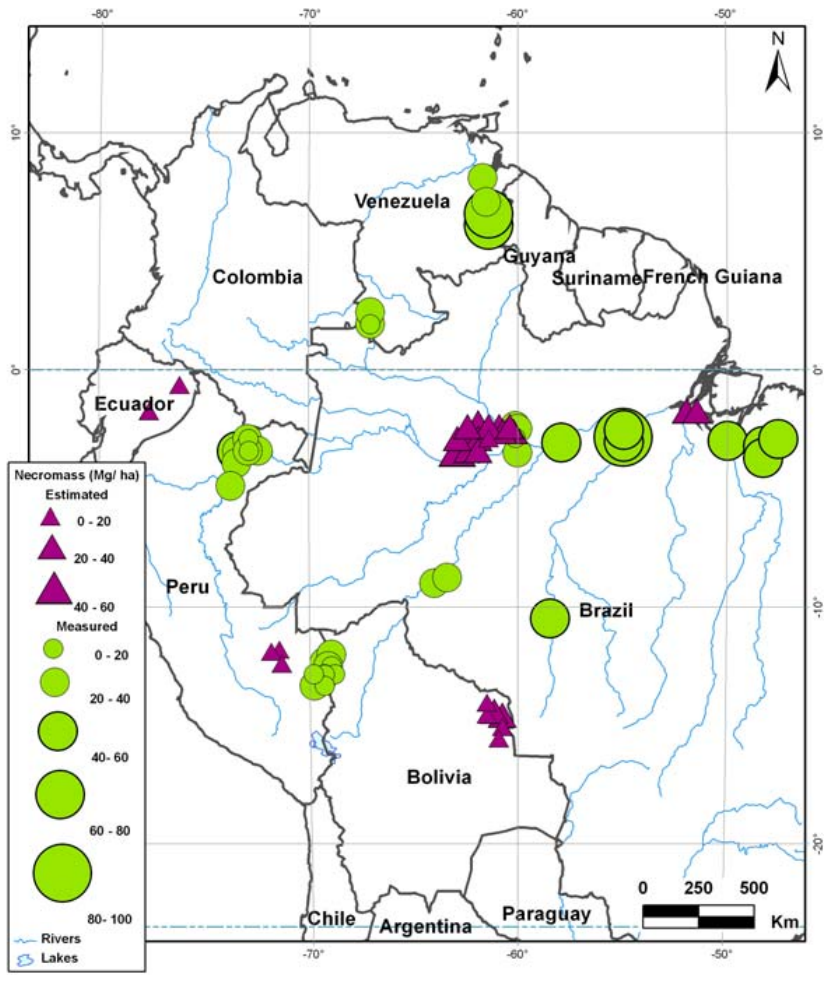

Fig. 2. Necromass in terra firma Amazonian forests. Estimated necromass (using Eq. 12, $n=27$ ), on the basis of known mortality mass inputs and plot-level wood density $\left(\rho_{B} j_{j}\right)$. Measured necromass (Table 2, $n=42$ ), on the basis of field measurement from this study and other published literature.

mortality, because trees with short-lifespans contribute less to the calculation (Lewis et al., 2004). However, when using different census lengths, it is very likely that the major trends will persist. Long-term studies of tree mortality dynamics across sites would provide a valuable extension to this study.

It is also arguable that in the cross site comparisons, the measured necromass $(N)$ values are not independent of plot-level living wood density $\left(\rho_{B A} j\right.$, Fig. 1c). These arguments may come from (1) $N$ was calculated as a function of CWD wood density $\left(\rho_{d}\right)$; (2) $\rho_{d}$ was derived from standaverage of wood density $\left(\rho_{B A}\right)$; (3) $\rho_{B A}$, and AGB were both derived from individual wood density $\left(\rho_{i}\right)$. It seems that correlations between these parameters are inevitable as they are founded on the same measures. However, parts of findings are based on other published, independently measured, terra firma data (Table 2). Also, when examining the relationship between $\rho_{B A j}$ and volume of CWD (independent of $\rho_{B A}$ ) across terra firma plots, the relationship between wood density and CWD stocks persists (linear regression, $p<0.001, r^{2}=0.389$, similar to that of Eq. 10). Together, these results confirm the strong gradient of decreasing CWD stocks from east to west parallel to a decrease in plot-level living wood density.

\subsection{Improving necromass estimation: method}

To account for the carbon store in coarse woody debris across Amazonia, current research (e.g. Houghton et al., 2001; Malhi et al., 2006; Saatchi et al., 2007) typically uses a simplified necromass/biomass ratio (0.091, reviewed in Houghton et al., 2001). However, the Houghton et al. (2001) result was not specifically applicable for humid forests and included a wide selection of forest types (e.g. abandoned pastures in Uhl et al., 1988; tropical dry forest in Delaney et al., 1997). Moreover, some eastern Amazonian studies showed an even higher ratio (e.g., 0.33 in Rice et al., 2004) that may result from past disturbances. Because necromass is a function of mortality and decomposition, and the link between necromass and biomass is indirect and tenuous, methods for estimating necromass stocks in Amazonia can be improved using a larger dataset based on dynamic measures.

Here, we apply the new dataset collected by this study to generate an updated estimation method for necromass across Amazonia. This is achieved by using a simple model from Olson (1963) and data in Table 2. Assuming forests are close to dynamic equilibrium, the decay rate $\left(k_{s s}\right)$ is a function of necromass and mortality mass input. Moreover, as decay rate is a function of wood density (Chambers et al., 2000), the estimated decay rates across Amazonian forests can be related to their plot-level average living wood density as:

$$
\begin{aligned}
k_{s s} & =I_{\text {recent }} / N=0.879\left[\rho_{B A}\right]^{2}-2.134\left[\rho_{B A}\right] \\
& +1.202\left(r^{2}=0.329, p=0.007\right)
\end{aligned}
$$

where $k_{s s}\left(\right.$ year $\left.^{-1}\right)$ is the estimated decay rate in steady state, derived from the ratio of recent mortality mass input rate ( $\left.I_{\text {recent }}\right)$ and necromass $(N) . \rho_{B A j}\left(\mathrm{~g} \mathrm{~cm}^{-3}\right)$ is the average living wood density, weighted by basal area, of plot $j$.

As a result, for a plot where necromass has not been measured, necromass can be predicted by their known mortality mass inputs and decay rate estimates:

$$
\begin{aligned}
N & =I_{\text {recent }} / k_{S S} \\
& =I_{\text {recent }} /\left(0.879\left[\rho_{B A j}\right]^{2}-2.134\left[\rho_{B A j}\right]+1.202\right)
\end{aligned}
$$

\subsection{Improving necromass estimation: estimated values based on RAINFOR data}

To demonstrate necromass estimates for places where necromass has not been measured, we applied Eq. (12) to estimate necromass stocks for all Amazonian terra firma permanent plots $(n=27)$ in a long-term research project (RAINFOR) that met four criteria: currently without necromass measurement, censused recently between 2000 and 2006, have short-term (3.5 to 6 years) mortality mass input data, and are included in a tree-by-tree database (Peacock et al., 2007) ( $n=27$, Appendix A). The estimated necromass values (Appendix A) together with measured necromass $(n=42$, Table 2$)$ showed a decreasing necromass gradient from north-eastern to southwestern Amazonia (Fig. 2). 
Table 3. Stocks of coarse necromass and coarse aboveground biomass of tropical rainforest ${ }^{\dagger}$ across Amazonian regions. $N$, necromass, the average $( \pm 1 \mathrm{SE})$ of measured $(n=42$, Table 2$)$ and estimated ( $n=27$, using Eq. 12$)$ necromass; AGB $_{\text {coarse, }}$ coarse aboveground biomass.

\begin{tabular}{|c|c|c|c|c|c|c|c|}
\hline \multirow[t]{2}{*}{ Region* } & \multirow{2}{*}{$\begin{array}{l}\text { Area } \\
\left(10^{6} \mathrm{~km}^{2}\right)^{\mathbb{q}}\end{array}$} & \multicolumn{2}{|c|}{ Average $\left(\mathrm{Mg} \mathrm{ha}^{-1}\right)$} & \multirow[b]{2}{*}{$N / A_{\text {G }}$ coarse } & \multicolumn{2}{|c|}{ Carbon stock $(\operatorname{Pg} C)^{\&}$} & \multirow[b]{2}{*}{ Total } \\
\hline & & $N$ & $\mathrm{AGB}_{\text {coarse }}$ & & $N$ & $\mathrm{AGB}_{\text {coarse }}$ & \\
\hline $\mathrm{NE}$ & 0.53 & $39.9 \pm 10.1$ & $328.9 \pm 42.8$ & $0.166 \pm 0.039$ & $1.1 \pm 0.3$ & $8.8 \pm 1.1$ & $9.8 \pm 1.4$ \\
\hline E & 3.95 & $36.0 \pm 2.7$ & $284.7 \pm 7.8$ & $0.132 \pm 0.013$ & $7.1 \pm 0.5$ & $56.3 \pm 1.5$ & $63.4 \pm 2.1$ \\
\hline NW & 0.76 & $24.5 \pm 2.6$ & $238.2 \pm 8.5$ & $0.103 \pm 0.011$ & $0.9 \pm 0.1$ & $9.0 \pm 0.3$ & $9.9 \pm 0.4$ \\
\hline SW & 0.42 & $17.5 \pm 1.8$ & $216.5 \pm 5.8$ & $0.082 \pm 0.009$ & $0.4 \pm 0.0$ & $4.6 \pm 0.1$ & $4.9 \pm 0.2$ \\
\hline S & 0.17 & $17.4 \pm 3.0$ & $206.7 \pm 17.4$ & $0.090 \pm 0.020$ & $0.1 \pm 0.0$ & $1.8 \pm 0.1$ & $1.9 \pm 0.2$ \\
\hline Total & 5.83 & & & & $9.6 \pm 1.0$ & $80.4 \pm 3.3$ & $90.0 \pm 4.3$ \\
\hline Average $^{\S}$ & & $33.0 \pm 3.0$ & $275.5 \pm 14.9$ & $0.127 \pm 0.010$ & & & \\
\hline
\end{tabular}

$\dagger$ Defined as all monthly mean temperature $\geq 18^{\circ} \mathrm{C}$ and $\leq 3$ dry months in FAO (2000). * NE: north-eastern (Venezuela, Guyana, Suriname, and French Guiana); NW: north-western (Columbia, Ecuador, and northern Peru); SW: south-western (Acre state of Brazil and southern Peru); S: southern (Bolivia); E: eastern (Brazil, excluding Acre). \& $\mathrm{C}$ stock is estimated as 50\% of mass (Elias and Potvin, 2003). II Area of tropical rainforest (FAO, 2000). ${ }^{\S}$ Weighted by the area of forest cover in each region.

\subsection{Improving necromass estimation: extrapolating across Amazonia}

To better estimate necromass stock across Amazonia, we scale up both the estimated and measured results of necromass regionally and then to the entire Amazonian basin. Firstly, both the estimated and measured results of necromass (Appendix A and Table 2) were averaged for each broadlydefined Amazonian region (Table 3), in order to estimate the regional necromass value. Secondly, we multiplied the averaged values of necromass by the "tropical rainforest" area (reported by FAO, 2000) in the same region (Table 3), in order to estimate total necromass for each region. The same method was applied for biomass. Finally, we assumed that coarse woody debris and living trees are 50\% carbon by dry weight (Elias and Potvin, 2003). Thus, across Amazonia, the estimated carbon stock ( $\pm 1 \mathrm{SE})$ in coarse necromass is 9.6 $\pm 1.0 \mathrm{PgC}$ and in coarse biomass is $80.4 \pm 3.3 \mathrm{PgC}$ (Table 3). Together, total coarse aboveground dead and alive coarse woody mass accounts for $90.0 \pm 4.3 \mathrm{Pg} \mathrm{C}$ (Table 3 ).

The quantity of coarse biomass estimated here is very similar to a result estimated by a kriging-based method (79.1 $\pm 19.6 \mathrm{Pg} \mathrm{C}$ ( $85 \%$ of the original reported value in Malhi et al. (2006) to account only for coarse wood), but much higher than a remote-sensing-based result $(56.3 \pm 9.6 \mathrm{Pg} \mathrm{C}$, Saatchi et al., 2007, adjusted by the $85 \%$ coarse wood factor). This may be partially explained by different methods for calculating forest cover. Moreover, the definition of forest types varied between studies: Saatchi et al. (2007) classified forests into terra firma, floodplain, and other vegetation types, whereas Malhi et al. (2006) and our study both used a broader definition of "tropical rainforest" defined by FAO (2000). Based on data from Table 2, the ratio of necromass to coarse biomass is different among forest types, sug- gesting that forest type can influence the quantity of CWD. Therefore, discounting the variation between different forest types could lead to either over- or underestimating mass stocks across Amazonia.

Our study has shown that necromass is not an invariant fraction of biomass, even within terra firma forests, nor is it invariant across regions (Kruskal-Wallis test, $p=0.021$, Table 3). Moreover, the average ratio weighted by area of each region $(0.127)$ is greater than the commonly used value (0.091) reported in Houghton et al. (2001). Future studies can either apply Eq. (12) for a plot-level necromass estimation, or apply the updated necromass/biomass ratios reported in Table 3 for regional estimations. To accurately measure aboveground carbon pools across Amazonia, further research should consider both the quantity of necromass in different forest types.

\section{Conclusions}

Other studies have demonstrated several macroecological gradients from east to west across Amazonia: increasing mortality rates (Phillips et al., 2004), and decreasing biomass (Baker et al., 2004; Malhi et al., 2006) and average wood density (Baker et al., 2004). This study reveals an additional gradient: that necromass decreases from east to west. Across terra firma forests in Amazonia, necromass stocks are related to both forest structural parameter (biomass), and forest dynamic parameters (the mortality mass input and decomposition surrogate (living wood density) of the same plot), partially supporting the predictions of our study. Moreover, necromass is better explained by forest dynamics than forest biomass. Coarse woody debris is a more significant component, relative to aboveground biomass, than most studies 
of large-scale carbon budgets have assumed, but conversely is substantially less important than ecological reports based in eastern Amazonia had suggested. Future research should focus on the long-term dynamics and controlling factors of decomposition of coarse woody debris, and explanations of the observed macroecological gradient.

\section{Appendix A}

\section{Estimated necromass of other RAINFOR plots}

Necromass of the RAINFOR plots $(n=27)$ estimated by applying the necromass Eq. (12). All plots are terra firma forests located in Amazonia, have been recensused recently between 2000 and 2006 (similar range with our measured $\mathrm{NE}$ and NW forests), and have short-term (3.5 to 6 years) mortality mass input data. All values are for trees $\geq 10 \mathrm{~cm}$ diameter.

\begin{tabular}{lllllllll}
\hline Region & $\begin{array}{l}\text { Plot } \\
\text { name }\end{array}$ & $\begin{array}{l}\text { Census } \\
\text { date }\end{array}$ & $\begin{array}{l}\text { Lati- } \\
\text { tude }\end{array}$ & $\begin{array}{l}\text { Longi- } \\
\text { tude }\end{array}$ & $\begin{array}{l}\text { Necro- } \\
\text { mass* }\end{array}$ & AGB $_{\text {coarse }}{ }^{\mathrm{I}}$ & $I_{\text {recent* }} \rho_{B A j^{*}}$ \\
\hline NW & SUM-01 & 2002.50 & -1.8 & -77.6 & 14.31 & 205.33 & 4.79 & 0.516 \\
NW & TIP-02 & 2002.09 & -0.6 & -76.2 & 14.17 & 181.06 & 3.77 & 0.574 \\
SW & ALM-01 & 2004.50 & -11.8 & -71.5 & 19.92 & 252.48 & 6.00 & 0.544 \\
SW & MNU-03 & 2001.70 & -11.9 & -71.4 & 16.06 & 184.51 & 5.61 & 0.505 \\
SW & MNU-04 & 2001.62 & -11.9 & -71.4 & 14.08 & 224.34 & 4.18 & 0.548 \\
S & CHO-01 & 2001.45 & -14.4 & -61.1 & 20.47 & 124.10 & 4.20 & 0.631 \\
S & CRP-01 & 2001.45 & -14.5 & -61.5 & 19.17 & 239.46 & 3.02 & 0.680 \\
S & CRP-02 & 2001.45 & -14.5 & -61.5 & 14.46 & 209.16 & 3.76 & 0.580 \\
S & HCC-21 & 2001.42 & -14.6 & -60.7 & 28.80 & 215.82 & 6.99 & 0.596 \\
S & HCC-22 & 2001.42 & -14.6 & -60.7 & 14.41 & 239.06 & 3.33 & 0.606 \\
S & LFB-01 & 2001.40 & -14.6 & -60.9 & 6.93 & 212.45 & 1.71 & 0.592 \\
E & BDF-03 & 2003.71 & -2.4 & -59.9 & 25.27 & 308.33 & 4.07 & 0.676 \\
E & BDF-04 & 2003.71 & -2.4 & -59.9 & 27.46 & 238.33 & 3.62 & 0.708 \\
E & BDF-05 & 2003.71 & -2.4 & -59.9 & 27.01 & 276.92 & 3.45 & 0.713 \\
E & BDF-06 & 2003.71 & -2.4 & -59.9 & 40.21 & 262.98 & 5.34 & 0.707 \\
E & BDF-07 & 2004.04 & -2.4 & -59.9 & 26.59 & 328.85 & 2.99 & 0.730 \\
E & BDF-08 & 2004.13 & -2.4 & -59.9 & 28.03 & 289.93 & 3.96 & 0.697 \\
E & BDF-09 & 2002.50 & -2.4 & -59.9 & 31.91 & 348.46 & 3.49 & 0.734 \\
E & BDF-10 & 2002.54 & -2.4 & -59.9 & 31.56 & 282.21 & 4.19 & 0.707 \\
E & BDF-11 & 2002.54 & -2.4 & -59.9 & 15.24 & 339.29 & 1.98 & 0.710 \\
E & BDF-12 & 2002.54 & -2.4 & -59.9 & 54.00 & 327.21 & 7.75 & 0.695 \\
E & BDF-13 & 2003.29 & -2.4 & -59.9 & 39.19 & 314.42 & 4.54 & 0.726 \\
E & BDF-14 & 2003.13 & -2.4 & -59.9 & 20.70 & 350.78 & 2.42 & 0.725 \\
E & CAX-01 & 2004.59 & -1.7 & -51.5 & 23.21 & 338.91 & 2.73 & 0.724 \\
E & CAX-02 & 2003.20 & -1.7 & -51.5 & 36.28 & 323.57 & 4.79 & 0.708 \\
E & JAC-01 & 2002.50 & -2.6 & -60.2 & 28.10 & 275.75 & 4.09 & 0.693 \\
E & JAC-02 & 2002.50 & -2.6 & -60.2 & 27.43 & 268.75 & 3.88 & 0.697 \\
\hline & & & & & & & &
\end{tabular}

$\dagger$ NE: north-eastern Amazonia (Venezuela, Guyana, Suriname, and French Guiana); NW: north-western Amazonia (Columbia, Ecuador, and northern Peru); SW: southwestern Amazonia (Acre state of Brazil and southern Peru); S: southern Amazonia (Bolivia); E: eastern Amazonia (Brazil, excluding Acre). * See Eq. (12), where Necromass is estimated necromass $\left(\mathrm{Mg} \mathrm{ha}^{-1}\right), I_{\text {recent }}$ is recent mortality mass input ( $\mathrm{Mg}$ $\mathrm{ha}^{-1}$ year $^{-1}$ ), and $\rho_{B A j}$ is the average living wood density of plot $j$, weighted by basal area. II coarse aboveground biomass $\left(\mathrm{Mg} \mathrm{ha}^{-1}\right)$, which is calculated by multiplying aboveground biomass $(\mathrm{AGB}, \mathrm{kg}$ ) with a correction factor $(0.85)$ to account for the proportion of biomass in branches $>10 \mathrm{~cm}$ diameter only (Higuchi, unpublished data, cited in Chambers et al., 2000). AGB is calculated by the equation in Chambers et al. (2001) and adjusted by species wood density (Baker et al., 2004).
Acknowledgements. This paper is a product of the RAINFOR network. RAINFOR is currently supported by the Gordon and Betty Moore Foundation. Valuable contributions were made in the field by Julio Serrano and Rodrigo Barros, Nestor Jaramillo, Isau Huamantupa Chuquimaco, Antonio Peña Cruz, and other field assistants. We thank Biodiversidad y Desarrollo Sustentable en Ecosistemas Forestales (BIODESUS), Instituto Nacional de Recursos Naturales (INRENA), Instituto de Investigaciones de la Amazonía Peruana (IIAP), and Explorama Tours S.A., for research permission and support. Fieldwork was supported by a Natural Environment Research Council grant and a Leverhulme Trust Research Fellowship to Oliver Phillips. The RAINFOR tree census database and wood density database are managed and compiled by Julie Peacock and Gabriela Lopez-Gonzalez. This research is part of the $\mathrm{PhD}$ study of Kuo-Jung Chao, supported by the Overseas Research Students Award (Universities UK), the School of Geography, and the University of Leeds. We benefited from helpful discussions with Lindsay Banin, Markus Wagner, and two referees. This research would not be possible through the pioneering work by Alwyn Gentry and Jean Pierre Veillon who established permanent plots in the neotropics.

Edited by: J. Lloyd

\section{References}

Baker, T. R., Phillips, O. L., Malhi, Y., Almeida, S., Arroyo, L., Di Fiore, A., Erwin, T., Killeen, T. J., Laurance, S. G., Laurance, W. F., Lewis, S. L., Lloyd, J., Monteagudo, A., Neill, D. A., Patiño, S., Pitman, N. C. A., Silva, J. N. M., and Vásquez Martínez, R.: Variation in wood density determines spatial patterns in Amazonian forest biomass, Global Change Biol., 10, 545-562, 2004.

Baker, T. R., Honorio Coronado, E. N., Phillips, O. L., Martin, J., van der Heijden, G. M. F., Garcia, M., and Silva Espejo, J.: Low stocks of coarse woody debris in a southwest Amazonian forest, Oecologia, 152, 495-504, 2007.

Brown, I. F., Martinelli, L. A., Thomas, W. W., Moreira, M. Z., Ferreira, C. A. C., and Victoria, R. A.: Uncertainty in the biomass of Amazonian forests: an example from Rondônia, Brazil, For. Ecol. Manag., 75, 175-189, 1995.

Carey, E. V., Brown, S., Gillespie, A. J. R., and Lugo, A. E.: Tree mortality in mature lowland tropical moist and tropical lower montane moist forests of Venezuela, Biotropica, 26, 255-265, 1994.

Chambers, J. Q., Higuchi, N., Schimel, J. P., Ferreira, L. V., and Melack, J. M.: Decomposition and carbon cycling of dead trees in tropical forests of the central Amazon, Oecologia, 122, 380$388,2000$.

Chambers, J. Q., dos Santos, J., Ribeiro, R. J., and Higuchi, N.: Tree damage, allometric relationships, and above-ground net primary production in central Amazon forest, For. Ecol. Manag., 152, 7384, 2001.

Chao, K.-J., Phillips, O. L., and Baker, T. R.: Wood density and stocks of coarse woody debris in a northwestern Amazonian landscape, Can. J. For. Res., 38, 795-825, 2008a.

Chao, K.-J., Phillips, O. L., Gloor, E., Monteagudo, A., TorresLezama, A., and Vásquez Martínez, R.: Growth and wood density predict tree mortality in Amazon forests, J. Ecol., 96, 281$292,2008 b$. 
Chave, J., Andalo, C., Brown, S., Cairns, M. A., Chambers, J. Q., Eamus, D., Fölster, H., Fromard, F., Higuchi, N., Kira, T., Lescure, J.-P., Nelson, B. W., Ogawa, H., Puig, H., Riéra, B., and Yamakura, T.: Tree allometry and improved estimation of carbon stocks and balance in tropical forests, Oecologia, 145, 8799, 2005.

Chave, J., Muller-Landau, H. C., Baker, T. R., Easdale, T. A., ter Steege, H., and Webb, C. O.: Regional and phylogenetic variation of wood density across 2456 neotropical tree species, Ecol. Appl., 16, 2356-2367, 2006.

Clark, D. A., Brown, S., Kicklighter, D. W., Chambers, J. Q., Thomlinson, J. R., Ni, J., and Holland, E. A.: Net primary production in tropical forests: an evaluation and synthesis of existing field data, Ecol. Appl., 11, 371-384, 2001.

Cummings, D. L., Kauffman, J. B., Perry, D. A., and Hughes, R. F.: Aboveground biomass and structure of rainforests in the southwestern Brazilian Amazon, For. Ecol. Manag., 163, 293-307, 2002.

Delaney, M., Brown, S., Lugo, A. E., Torres-Lezama, A., and Bello Quintero, N.: The distribution of organic carbon in major components of forests located in five life zones of Venezuela, J. Ecol., 13, 697-708, 1997.

Delaney, M., Brown, S., Lugo, A. E., Torres-Lezama, A., and Quintero, N. B.: The quantity and turnover of dead wood in permanent forest plots in six life zones of Venezuela, Biotropica, 30, 2-11, 1998.

Elias, M. and Potvin, C.: Assessing inter- and intra-specific variation in trunk carbon concentration for 32 neotropical tree species, Can. J. For. Res., 33, 1039-1045, 2003.

FAO: Global Forest Resources Assessment (Food and Agriculture Organization of the United Nations), http://www.fao.org/ forestry/site/fra2000report/en/, access: 27 September 2005, 2000.

Gale, N.: The aftermath of tree death: coarse woody debris and the topography in four tropical rain forests, Can. J. For. Res., 30, 1489-1493, 2000.

Gerwing, J. J.: Degradation of forests through logging and fire in the eastern Brazilian Amazon, For. Ecol. Manag., 157, 131-141, 2002.

Harmon, M. E., Franklin, J. F., Swanson, F. J., Sollins, P., Gregory, S. V., Lattin, J. D., Anderson, N. H., Cline, S. P., Aumen, N. G., Sedell, J. R., Lienkaemper, G. W., Cromack, K. J., and Cummins, K. W.: Ecology of coarse woody debris in temperate ecosystems, Adv. Ecol. Res., 15, 133-302, 1986.

Harmon, M. E. and Sexton, J.: Guidelines for Measurements of Woody Detritus in Forest Ecosystems, US Long Term Ecological Research Network Office Publication Seattle, Washington, 1996.

Higuchi, N., Santos, J. D., Ribeiro, R. J., Minette, L., and Biot, Y.: Biomassa da parte aérea da vegetação da floresta tropical úmida de terra-firme da Amazônia Brasileira, Acta Amazonica, 28, 153-166, 1998.

Houghton, R. A., Lawrence, K. T., Hackler, J. L., and Brown, S.: The spatial distribution of forest biomass in the Brazilian Amazon: a comparison of estimates, Global Change Biol., 7, 731746, 2001.

Kauffman, J. B., Uhl, C., and Cummings, D. L.: Fire in the Venezuela Amazon 1: Fuel biomass and fire chemistry in the evergreen rainforest of Venezuela, Oikos, 53, 167-175, 1988.

Keller, M., Palace, M., Asner, G. P., Pereira, R., and Silva, J. N. M.:
Coarse woody debris in undisturbed and logged forests in the eastern Brazilian Amazon, Global Change Biol., 10, 784-795, 2004.

Klinge, H.: Biomassa y materia orgánica del suelo in el ecosistema de la pluviselva centro-amazonica, Acta Cient. Venez., 24, 174181, 1973.

López-Gonzalez, G., Baker, T. R., Lewis, S. L., Peacock, J., and Phillips, O. L.: Functional Trait Database, RAINFOR (http: //www.geog.leeds.ac.uk/projects/rainfor/), date of extraction: 8 February 2007, 2006.

Lewis, S. L., Phillips, O. L., Sheil, D., Vinceti, B., Baker, T. R., Brown, S., Graham, A. W., Higuchi, N., Hilbert, D. W., Laurance, W. F., Lejoly, J., Malhi, Y., Monteagudo, A., Núñez Vargas, P., Sonke, B., Supardi, M. N. N., Terborgh, J. W., and Vásquez Martínez, R.: Tropical forest tree mortality, recruitment and turnover rates: calculation, interpretation and comparison when census intervals vary, J. Ecol., 92, 929-944, 2004.

Malhi, Y., Phillips, O. L., Lloyd, J., Baker, T. R., Wright, J., Almeida, S., Arroyo, L., Frederiksen, T., Grace, J., Higuchi, N., Killeen, T., Laurance, W. F., Leaño, C., Lewis, S. L., Meir, P., Monteagudo, A., Neill, D., Núñez Vargas, P., Panfil, S. N., Patiño, S., Pitman, N., Quesada, C. A., Rudas-Ll., A., Salomão, R., Saleska, S., Silva, N., Silveira, M., Sombroek, W. G., Valencia, R., Vásquez Martínez, R., Vieira, I. C. G., and Vinceti, B.: An international network to monitor the structure, composition and dynamics of Amazonian forests (RAINFOR), J. Veg. Sci., 13, 439-450, 2002.

Malhi, Y., Wood, D., Baker, T. R., Wright, J., Phillips, O. L., Cochrane, T., Meir, P., Chave, J., Almeida, S., Arroyo, L., Higuchi, N., Killeen, T. J., Laurance, S. G., Laurance, W. F., Lewis, S. L., Monteagudo, A., Neill, D. A., Núñez Vargas, P., Pitman, N. C. A., Quesada, C. A., Salomão, R., Silva, J. N. M., Torres Lezama, A., Terborgh, J., Vásquez Martínez, R., and Vinceti, B.: The regional variation of aboveground live biomass in oldgrowth Amazonian forests, Global Change Biol., 12, 1107-1138, 2006.

Martius, C.: Decomposition of wood, in: The Central Amazon Floodplain - Ecology of a Pulsing System, edited by: Junk, W. J., Springer, Germany, 267-276, 1997.

Martius, C. and Bandeira, A. G.: Wood litter stocks in tropical moist forest in central Amazonia, Ecotropica, 4, 115-118, 1998.

Nascimento, H. E. M. and Laurance, W. F.: Total aboveground biomass in central Amazonian rainforests: a landscape-scale study, For. Ecol. Manag., 168, 311-321, 2002.

Olson, J. S.: Energy storage and the balance of producers and decomposers in ecological systems, Ecology, 44, 322-331, 1963.

Palace, M., Keller, M., and Silva, H.: Necromass production: studies in undisturbed and logged Amazon forests, Ecol. Appl., 18, 873-884, 2008.

Palace, M. W., Keller, M., Asner, G., Silva, J. N. M., and Passos, C.: Necromass in undisturbed and logged forests in the Brazilian Amazon, For. Ecol. Manag., 238, 309-318, 2007.

Peacock, J., Baker, T. R., Lewis, S. L., López-Gonzalez, G., and Phillips, O. L.: The RAINFOR plot database: monitoring forest biomass and dynamics, J. Veg. Sci., 18, 535-542, 2007.

Phillip, M. S.: Measuring Trees and Forests, 2nd ed., CAB International, Wallingford, UK, 1994.

Phillips, O. L., Baker, T. R., Arroyo, L., Higuchi, N., Killeen, T. J., Laurance, W. F., Lewis, S. L., Lloyd, J., Malhi, Y., Monteagudo, 
A., Neill, D. A., Núñez Vargas, P., Silva, J. N. M., Terborgh, J., Vásquez Martínez, R., Alexiades, M., Almeida, S., Brown, S., Chave, J., Comiskey, J. A., Czimczik, C. I., Di Fiore, A., Erwin, T., Kuebler, C., Laurance, S. G., Nascimento, H. E. M., Olivier, J., Palacios, W., Patiño, S., Pitman, N. C. A., Quesada, C. A., Saldias, M., Torres Lezama, A., and Vinceti, B.: Pattern and process in Amazon tree turnover, 1976-2001, Philos. T. Roy. Soc. B., 359, 381-407, 2004.

RAINFOR: Amazon Forest Inventory Network, http://www.geog. leeds.ac.uk/projects/rainfor/, access: 8 February 2007.

Rice, A. H., Hammond Pyle, E., Saleska, S. R., Hutyra, L., de Camargo, P. B., Portilho, K., Marques, D. F., and Wofsy, S. C.: Carbon balance and vegetation dynamics in an old-growth Amazonian forest, Ecol. Appl., 14(4) suppl., S55-S71, 2004.

Saatchi, S. S., Houghton, R. A., Dos Santos Alvalá, R. C., Soares, J. V., and Yu, Y.: Distribution of aboveground live biomass in the Amazon basin, Global Change Biol., 13, 816-837, 2007.

Saldarriaga, J. G., West, D. C., Tharp, M. L., and Uhl, C.: Longterm chronosequence of forest succession in the upper Rio Negro of Colombia and Venezuela, J. Ecol., 76, 938-958, 1988.
Scott, D. A., Proctor, J., and Thompson, J.: Ecological studies on a lowland everygreen rain forest on Maracá Island, Roraima, Brasil. II. Litter and nutrient cycling, J. Ecol., 80, 705-717, 1992.

Summers, P. M.: Estoque, decomposição, e nutrientes da liteira grossa em floresta de terra-firme, na Amazônia central, MS, Instituto Nacional de Pesquisas da Amazônia, Manaus, Brazil, 1998.

Uhl, C., Buschbacher, R., and Serrão, E. A. S.: Abandoned pastures in eastern Amazônia, I: patterns and succession, J. Ecol., 76, 663-681, 1988.

Uhl, C. and Kauffman, J. B.: Deforestation, fire susceptibility, and potential tree responses to fire in the eastern Amazon, Ecology, 71, 437-449, 1990.

Vásquez Martínez, R.: Flórula de las Reservas Biológicas de Iquitos, Perú, Missouri Botanical Garden, St. Louis, 1997.

Vásquez Martínez, R. and Phillips, O. L.: Allpahuayo: floristics, structure, and dynamics of a high-diversity forest in Amazonian Peru, Annals Missouri Botanical Garden, 87, 499-527, 2000.

Veillon, J. P.: El crecimiento de algunos bosques naturales de Venezuela en relación con los parámetros del medio ambiente, Revista Forestal Venezolana, 29, 5-120, 1985. 